\title{
Personal reflections on RNA: an emphasis on trypanosomes
}

\section{KEN STUART}

Seattle Biomedical Research Institute, Seattle, Washington 98109, USA
I was drawn into the field of RNA biology as a graduate student interested in the regulation of cellular processes in pathogenic protozoa. I thought that the mechanisms that African Trypanosomes use to control the differential expression of their mitochondrial (mt) genes during their life cycle must be fundamentally important and critical to this lethal pathogen. This is because these pathogens generate energy generation by oxidative phosphorylation in the insect vector but by glycolysis in the mammalian host. My naïve expectation was that this would entail conventional mechanisms such as regulation of transcription initiation to switch between the different modes of energy generation. I was surprised when it turned out to be the novel process of RNA editing, by which the coding sequences of primary mitochondrial transcripts are changed by the insertion and deletion of Us. When sequencing the unusual $\mathrm{mt}$ genome of $T$. brucei we found a frameshift in the COII gene, as did the Benne and Simpson groups in related protozoa. Benne showed that four U insertions in the mRNA eliminated the frameshift. Shortly thereafter we showed that numerous $U$ insertions extended the $\mathrm{N}$-terminal coding sequence of Cyb mRNA and created an initiation codon but this only occurred in the insect stage. Additional mRNA sequencing revealed extensive editing of COIII mRNA by the insertion and deletion, respectively, of hundreds and tens of Us. Extensive sequencing of $\mathrm{mt}$ mRNAs of T. brucei in my lab and of the related protozoa in other labs showed different edited $\mathrm{mt}$ mRNAs in these species, which is most extensive in T. brucei. Overall editing creates initiation and termination codons and creates or extends open reading frames in multiple mt mRNAs. We demonstrated translation products of the edited mRNAs by mass spectrometry showing that they are translated. Some edited mRNAs like Cyb are abundant in the insect stage, others in the mammalian stage and still others in both stages. This pattern of the differential mRNA editing matches the different energy generating processes between the life cycle stages. However, the regulatory mechanism that controls the differential editing is a mystery.

Our initial reports of RNA editing were met with skepticism, especially when I presented the extensive editing results

\footnotetext{
Corresponding author: ken.stuart@seattlebiomed.org

Article and publication date are at http://www.rnajournal.org/cgi/doi/10. 1261/rna.050195.115. Freely available online through the RNA Open Access option.
}

at a Cold Spring Harbor RNA processing meeting. I was invited at the last minute and given no details but told when I arrived on the first night that I would have $5 \mathrm{~min}$ at the end of the morning session. It was daunting because of many renowned RNA scientists in the audience and the excellent detailed presentations that preceded mine. My presentation was followed by $30 \mathrm{~min}$ of questions and lunch was abuzz with disbelief. The skepticism was later dispelled by additional work by several groups and especially by the discovery of guide RNAs (gRNAs) by Beat Blum in the Simpson lab. The gRNAs are encoded in the unusual minicircle component of trypanosome mtDNA. The gRNAs have $3^{\prime}$ oligo(U) tails and are complementary to the edited sequences, which immediately suggested that they specify the edited sequence. We had sequenced a chimeric gRNA/edited mRNA molecule but did not recognize the complementarity to the edited sequence or its minicircle source until we saw Blum's results. The thousands of minicircles in T. brucei each encode multiple gRNAs, which amply account for extensive editing. We then focused on the mechanism of editing. Two proposed mechanisms suggested that the Us were derived from or transferred to the gRNA $U$ tail via a gRNA/mRNA chimeric intermediate; they differed by whether this was accomplished by transesterification or by enzymatic cleavage and ligation steps. We showed that editing occurred by the third proposed mechanism, namely by rounds of enzymatic cleavage, $U$ addition or removal, and ligation. Stimulated by presentations at a RNA society meeting we developed an in vitro editing system and demonstrated key elements of the editing process including the requirements for mt extract, specific gRNAs, ATP and UTP for addition but not deletion. We found that chimeras are not intermediates but are byproducts that are rare in vivo. The role of the gRNA $U$ tail is uncertain. It is not essential for in vitro editing but in vivo it may stabilize the gRNA/mRNA association during editing or participate in regulating specific editing steps.

We found that editing is catalyzed by complexes that sediment at $\sim 20$ S and contain 21 proteins. Aswini Panigrahi purified the complexes and we identified the proteins by MS. The catalytic functions were predicted from their sequence motifs and verified by various in vitro and in vivo

(C) 2015 Stuart This article, published in $R N A$, is available under a Creative Commons License (Attribution-NonCommercial 4.0 International), as described at http://creativecommons.org/licenses/by-nc/4.0/. 
experiments. I was initially uncertain of the predictive value of the motifs but gratified that they were all accurate. We showed that the complexes contain three endonucleases, a $3^{\prime}$ terminal uridylyl transferase, two $3^{\prime}$ uridylyl exonucleases and two RNA ligases. They also contained 13 proteins with no apparent functional catalytic motifs, but which fall into two general groups; seven with an N-terminal U1 Zn finger motif and six with a C-terminal OB-fold. We determined interactions between proteins and the overall general organization and found that the complexes contain stable heterotrimeric insertion and deletion subcomplexes, which can, respectively, catalyze the $\mathrm{U}$ insertion or deletion and ligation steps that follow the endonucleolytic cleavage, which initiates each round of editing. Jason Carnes and others in the lab showed that there are three types of complexes that differ by which of three endonucleases and partner protein pairs that they contain, and by their cleavage specificity. These complexes have a common core of the heterotrimers and six other proteins that specifically interact. Two other proteins appear to transiently interact with the complex. The complexes discriminate among, and specifically cleave, the thousands of editing sites in T. brucei. The editing of these numerous sites is extremely precise, which may be attained by specific recognition and cleavage of mRNA/gRNA heteroduplex mismatches by the endonucleases, aided by their partner proteins and perhaps other factors. The mRNA editing proceeds $3^{\prime}$ to $5^{\prime}$ due to the antiparallel interaction between the $5^{\prime}$ sequence of the cognate gRNAs that is complementary to mRNA sequence $3^{\prime}$ to sequence edited by the gRNA. Trypanosome $\mathrm{mt}$ contain numerous partially edited mRNAs that were likely being editing when isolated. They have an incompletely edited sequence between their $3^{\prime}$ edited and $5^{\prime}$ unedited regions with characteristics that suggests editing by the different complexes, re-editing and that editing does not invariably proceed precisely $3^{\prime}$ to $5^{\prime}$. The complexes must somehow collaborate to edit the insertion and deletion that is specified by single gRNA. They must also function dynamically to relocate the editing sites and the catalytic centers relative to each other and interact with other molecules, proteins and complexes that function in or regulate the editing. RNA editing is integrated within the overall $\mathrm{mt} \mathrm{RNA}$ processing, which employs the functions of other proteins and complexes. Indeed, the regulation of differential editing is integrated with other cellular processes as illustrated by it being coordinated with other developmental processes.

Other RNA editing was discovered shortly after the initial findings of RNA editing in trypanosomes, which I would like to think that our work helped stimulate. The types of cellular and viral RNAs, the mechanisms of their editing, and the functional consequences are as varied as is their biological distribution, which ranges from organellar RNAs to mammals. The biological functions that are affected by RNA editing span translation and metabolism through vertebrate development, neuronal function and behavior. A next frontier in the study in RNA editing may be elucidating the reg- ulatory processes. It is likely that these regulatory processes will be integrated within multiple molecular and cellular processes. Their elucidation may require a systems biology approach and may illuminate new biological paradigms.

Working on parasitic protozoa led to other RNA adventures. Some stemmed from my participation in the projects that first sequenced and annotated the genomes of three related protozoan parasites, each of which encodes about 10,000 proteins. It was arduous and costly at the time but surprisingly showed that the genomes of these early diverged eukaryotes are organized with polygenic clusters that are transcribed from a modest number of Pol II promoters. The polygenic transcripts are processed by trans-splicing (which others found also occurs in other organisms, e.g., nematodes) and polyadenylation. Consequently the abundance of almost all mRNAs is controlled post-transcriptionally in trypanosomes. An exception is the telomeric expression sites (ESs), which encode the variant surface glycoproteins (VSGs) and a few other genes, and which are transcribed by Pol I. Importantly, the genome has $\sim 20$ ESs and 2000 VSG genes and pseudogenes at other genomic sites but only one ES is transcribed at a time, and only in mammalian stages. The parasites periodically change which VSG gene is expressed by switching transcription to another telomeric site or by changing the VSG sequence by recombination. This results in antigenic variation and evasion of the host immune responses. My group, and the Agabian, Borst and Cross groups, characterized the processes of antigenic variation but were unable to identify the regulatory mechanisms that control the ES transcription. Recently, Igor Cestari in my lab found that the inositol pathway controls both monoallelic transcription of the ES and antigenic switching. This pathway, through its metabolites, may also control ES transcription between life cycle stages and coordinate multiple cellular processes. Thus, understanding antigenic variation led to understanding the control of RNA transcription, which in this case is by epigenetic control of transcription elongation.

We discovered also double stranded RNA viruses in Leishmania and saw a loose association between strains that have the virus and pathogenesis in humans. We stopped these studies because we could not experimentally manipulate and transmit the viruses. Years later others found that the virus appears to affect Leishmania virulence apparently via interaction with Toll-like receptors. Others also found a correlation between the presence of the RNAi machinery and Leishmania strains that contain the virus. Recently, I began studies of human immune cell transcriptome responses to malaria infection and vaccination and thus am again being driven (willingly) toward RNA analyses.

I have found the study of RNA to be fascinating, exciting and illuminating and suspect there are more surprises to come. They may arise from studies of regulatory processes, which are controlled by or act on RNA, or from analyses of multimolecular complexes that interact with RNA. The RNA era is not over. 

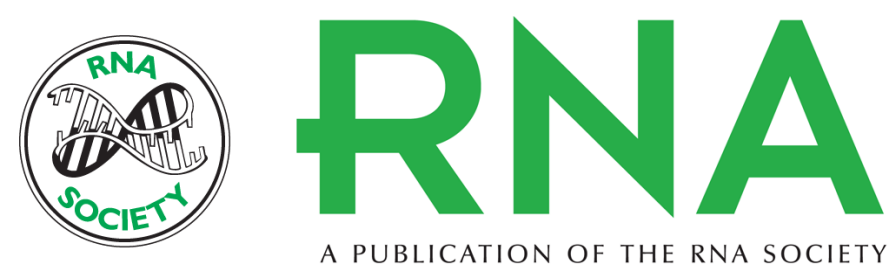

A PUBLICATION OF THE RNA SOCIETY

\section{Personal reflections on RNA: an emphasis on trypanosomes}

Ken Stuart

RNA 2015 21: 745-746
Open Access Freely available online through the RNA Open Access option.
Creative This article, published in RNA, is available under a Creative Commons License Commons (Attribution-NonCommercial 4.0 International), as described at License http://creativecommons.org/licenses/by-nc/4.0/.

Email Alerting Receive free email alerts when new articles cite this article - sign up in the box at the Service top right corner of the article or click here.

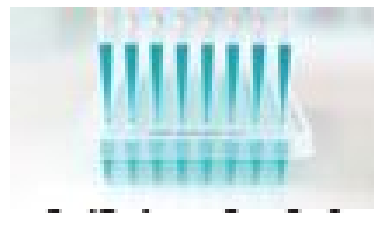

\section{Providing Precise Solutions for} your research.

To subscribe to RNA go to:

http://rnajournal.cshlp.org/subscriptions

(C) 2015 Stuart; Published by Cold Spring Harbor Laboratory Press for the RNA Society 